$\square$ Math-Net.Ru

Общероссийский математический портал 
О. Ю. Шведов, О пространстве Фока для систем с линейными связями, Матем. заметки, 2005, том 78, выпуск 1, 151156

DOI: https://doi.org/10.4213/mzm2572

Использование Общероссийского математического портала Math-Net.Ru подразумевает, что вы прочитали и согласны с пользовательским соглашением http://www. mathnet.ru/rus/agreement 
Параметры загрузки:

IP: 54.164 .48 .24

26 апреля 2023 г., 15:02:26

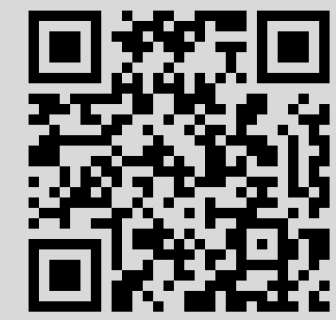




\section{О ПРОСТРАНСТВЕ ФОКА ДЛЯ СИСТЕМ С ЛИНЕЙНЫМИ СВЯЗЯМИ}

\section{О. Ю. Шведов}

1. В квантовой теории поля часто встречаются системы со связями: все калибровочные теории относятся к данному типу [1]. Один из возможных способов учесть связи, используемых в теоретической физике, заключается в том, чтобы модифицировать скалярное произведение в пространстве состояний следующим образом [2]:

$$
((\Psi, \Psi))=\left(\Psi, \prod_{a} \delta\left(\widehat{\Lambda}_{a}\right) \Psi\right)
$$

где $\widehat{\Lambda}_{a}$ - операторы связей.

Работа выполнена при финансовой поддержке Российского фонда фундаментальных исследований, грант № 02-01-01062.

(C) О.Ю. Шведов 
В сообщении исследуются свойства пространства Фока со скалярньп произведением (1) в случае, если связи $\widehat{\Lambda}_{a}$ линейны по операторам рождения и уничтожения, а также свойства операторов эволюции $e^{-i H t}$ в этом пространстве для случая оператора Гамильтона $H$, квадратичного по операторам рождения и уничтожения.

2. Пусть $\mathcal{H}$ - комплексное гильбертово пространство. Напомним, что бозонныл пространством Фока $\mathscr{F}(\mathscr{H})$ называется [3] прямая сумма

$$
\bigoplus_{n=0}^{\infty} \operatorname{Sym} \mathscr{H}^{\otimes n}
$$

вектор вида $\left(1 \in \mathscr{H}^{\otimes 0}, 0,0, \ldots\right) \equiv|0\rangle$ называется вакуумным вектором, операторы рождения и уничтожения $a^{ \pm}[f](f \in \mathscr{H})$ вводятся по формулам

$$
\begin{aligned}
& a^{-}[f] \operatorname{Sym} f_{1} \otimes \cdots \otimes f_{n}=\frac{1}{\sqrt{n}} \sum_{i=1}^{n}\left(f, f_{i}\right) \operatorname{Sym} f_{1} \otimes \cdots \otimes f_{i-1} \otimes f_{i+1} \otimes \cdots \otimes f_{n}, \\
& a^{+}[f] \operatorname{Sym} f_{1} \otimes \cdots \otimes f_{n}=\sqrt{n+1} \operatorname{Sym} f \otimes f_{1} \otimes \cdots \otimes f_{n} .
\end{aligned}
$$

Через $S \subset \mathscr{F}(\mathscr{H})$ обозначим множество векторов $\Psi=\left(\Psi_{0}, \Psi_{1}, \ldots\right)$ таких, что

$$
\|\Psi\|_{m}=\max _{n} n^{m}\left\|\Psi_{n}\right\|<\infty, \quad m=0,1,2, \ldots
$$

Нормы (2) задают топологию в $S$.

3. Рассмотрим $\mathscr{H}_{\mathbb{R}}$ - овеществление гильбертова пространства $\mathscr{H}$ - множество, совпадающее с $\mathscr{H}$, на котором введена операция умножения на вещественное число и скалярное произведение $(f, g)_{\mathbb{R}}=\operatorname{Re}(f, g)$. Введем на $\mathscr{H}_{\mathbb{R}}$ следующие структуры: операторнозначную 1-форму $\Omega[f]=-i\left(a^{+}[f]-a^{-}[f]\right)$ и билинейную форму $\langle f, g\rangle=i((f, g)-(g, f))$. При этом $[\Omega[f] ; \Omega[g]]=$ $-i\langle f, g\rangle$.

Введем скалярное произведение на $S$ следующим образом. Пусть $\mathscr{L}_{k}$ - конечномерное $(k$-мерное) подпространство $\mathscr{H}_{\mathbb{R}}$, удовлетворяющее свойству $\left\langle\varphi, \varphi^{\prime}\right\rangle=0$ при всех $\varphi, \varphi^{\prime} \in \mathscr{L}_{k}$, с мерой $d \mu(\varphi)$, инвариантной относительно сдвигов. Положим

$$
\left(\left(\Psi^{\prime}, \Psi\right)\right)=\int_{\mathscr{L}_{k}} d \mu(\varphi)\left(\Psi^{\prime}, e^{i \Omega[\varphi]} \Psi\right), \quad \Psi, \Psi^{\prime} \in S .
$$

Если выбрать базис $\varphi^{(1)}, \ldots, \varphi^{(k)}$ на $\mathscr{L}_{k}$ и ввести на $\mathscr{L}_{k}$ координаты по формуле $\varphi=$ $\sum_{a} \alpha_{a} \varphi^{(a)}$, то мера $d \mu(\varphi)$ запишется как $J d \alpha_{1} \ldots d \alpha_{k}$, а формула (3) в виде

$$
\left(\left(\Psi^{\prime}, \Psi\right)\right)=J \int d \alpha_{1} \cdots d \alpha_{k}\left(\Psi^{\prime}, e^{i \sum_{a} \alpha_{a} \Omega\left[\varphi^{(a)}\right]} \Psi\right)
$$

совпадающем с (1).

Теорема 1. При $\Psi, \Psi^{\prime} \in S$ интеграл (3) для скалярного произведения сходится. При этом $\left(\left(\Psi^{\prime}, \Psi\right)\right)$ непрерывно по $\Psi, \Psi^{\prime}$ в топологии $S,((\Psi, \Psi)) \geqslant 0$, a для любых $\Psi, \Psi^{\prime} \in S$ при $\varphi \in \mathscr{L}_{k}$ справедливо свойство $\left(\left(\Psi^{\prime}, \Omega[\varphi] \Psi\right)\right)=0$.

Положим $\Psi \sim 0$, если $((\Psi, \Psi))=0$. Пополняя факторпространство $S / \sim$, получаем гильбертово пространство $\bar{S}=\overline{S / \sim}$, которое и рассматривается в качестве пространства состояний для системы со связями $\Omega\left[\varphi^{(a)}\right]$. Назовем его пространством Фока для системы с линейньми связями. Некоторые свойства операторов в $S$, линейных по $a^{ \pm}$, дает следующая теорема.

TEOPEMA 2. Пусть $Y \in \mathscr{H}_{\mathbb{R}} u Y\langle\perp\rangle \mathscr{L}_{k}$. Тогда оператор $\Omega(Y)$ эрмитов на $S$ и сохраняет отношение эквивалентности; оператор $e^{i \Omega(Y)}$ определен на $S$, сохраняет норму отношение эквивалентности, однозначно продолжается до унитарного оператора в $\bar{S}$. 
ЗАмечаниЕ. Если $Y$ не косоортогонален $\mathscr{L}_{k}$, то $\Omega(Y)$ не сохраняет отношение эквивалентности на $S$.

4. Сопоставим каждому $A \in \operatorname{Sym} \mathscr{H} \otimes \mathscr{H}$ и $A^{*} \in \mathrm{Sym} \mathscr{H}^{*} \otimes \mathscr{H}^{*}$ операторы $\frac{1}{2} a^{+} A a^{+}: S \rightarrow S$, $\frac{1}{2} a^{-} A^{*} a^{-}: S \rightarrow S, \widehat{A}: \mathscr{H}^{*} \rightarrow \mathscr{H}, \widehat{A}^{*}: \mathscr{H} \rightarrow \mathscr{H}^{*}$, положив при $A=\sum_{i j} A_{i j} f_{i} \otimes f_{j}, A^{*}=$ $\sum_{i j} A_{i j}^{*} f_{i}^{*} \otimes f_{j}^{*}, A_{i j}=A_{j i}, A_{i j}^{*}=A_{j i}^{*}$

$$
\begin{aligned}
& \frac{1}{2} a^{+} A a^{+} \equiv \frac{1}{2} \sum_{i j} A_{i j} a^{+}\left[f_{i}\right] a^{+}\left[f_{j}\right], \quad \widehat{A} \varphi^{*} \equiv \sum_{i j} M_{i j} \varphi^{*}\left[f_{i}\right] f_{j} \in \mathscr{H}, \\
& \frac{1}{2} a^{-} A^{*} a^{-} \equiv \frac{1}{2} \sum_{i j} A_{i j}^{*} a^{-}\left[f_{i}\right] a^{-}\left[f_{j}\right], \quad \widehat{A}^{*} \varphi \equiv \sum_{i j} A_{i j}^{*} \varphi^{*}\left[f_{i}\right] f_{j} \in \mathscr{H} \text {. }
\end{aligned}
$$

Сопоставим самосопряженному оператору $\widehat{B}: \mathscr{H} \rightarrow \mathscr{H}$ оператор $a^{+} \widehat{B} a^{-}$в $S$ вида $a^{+} \widehat{B} a^{-} \operatorname{Sym} f_{1} \otimes \cdots \otimes f_{n} \equiv \sum_{i=1}^{n} \operatorname{Sym} f_{1} \otimes \cdots \otimes f_{i-1} \otimes \widehat{B} f_{i} \otimes f_{i+1} \otimes \cdots \otimes f_{n}, \quad f_{s} \in D(\widehat{B})$.

Известно [3], что выражение

$$
\Psi_{M}=\exp \left[\frac{1}{2} a^{+} M a^{+}\right]|0\rangle
$$

определяет вектор из $S$ при $\|\widehat{M}\|<1$. Будем назьвать вектор (4) гауссовским вектором.

Обобщим на системы со связями понятие комплексного ростка Маслова [4], [5], отвечающего гауссовскому вектору (4). Введем некоторые вспомогательные понятия.

Рассмотрим $\mathscr{H}_{\mathbb{R} C} \equiv \mathscr{H}_{\mathbb{R}} \otimes_{\mathbb{R}} \mathbb{C}$-комплексификацию $\mathscr{H}_{\mathbb{R}}$, а также изоморфизм $\mathscr{H}_{\mathbb{R C}} \rightarrow \mathscr{H} \oplus \mathscr{H}^{*}$ вида

$$
f^{\prime} \otimes_{\mathbb{R}} 1+f^{\prime \prime} \otimes_{\mathbb{R}} i \mapsto\left(\begin{array}{c}
f^{\prime}+i f^{\prime \prime}=f \in \mathscr{H} \\
f^{\prime *}+i f^{\prime \prime *}=\varphi^{*} \in \mathscr{H}^{*}
\end{array}\right)
$$

В дальнейшем будем отождествлять элементы $\mathscr{H}_{\mathbb{R C}}$ и упорядоченные пары

$$
\left(\begin{array}{cl}
f & \in \mathscr{H} \\
\varphi^{*} & \in \mathscr{H}^{*}
\end{array}\right)
$$

Все операции, введенные на $\mathscr{H}_{\mathbb{R}}$, переносятся на $\mathscr{H}_{\mathbb{R} C} \cdot$ Их явный вид следующий.

Лемма 3. В новых обозначениях

$$
\begin{gathered}
\left(\begin{array}{c}
f \\
\varphi^{*}
\end{array}\right)^{*}=\left(\begin{array}{c}
\varphi \\
f^{*}
\end{array}\right), \quad\left(\left(\begin{array}{c}
f \\
\varphi^{*}
\end{array}\right),\left(\begin{array}{c}
g \\
\chi^{*}
\end{array}\right)\right)=\frac{1}{2}\left[(f, g)+\left(\varphi^{*}, \chi^{*}\right)\right] \\
\left\langle\left(\begin{array}{c}
f \\
\varphi^{*}
\end{array}\right),\left(\begin{array}{c}
g \\
\chi^{*}
\end{array}\right)\right\rangle=i\left[\varphi^{*}[g]-\chi^{*}[f]\right], \quad \Omega\left[\left(\begin{array}{c}
f \\
\varphi^{*}
\end{array}\right)\right]=-i\left(a^{+}[f]-a^{-}[\varphi]\right) .
\end{gathered}
$$

Назовем комплексным $S$-ростком Маслова $r(M)$, отвечающим гауссовскому вектору (4), множество всех таких $\chi \in \mathscr{H}_{\mathbb{R C}}$, что $\Omega[\chi] \Psi_{M}=0$ в смысле $S ;$ жомплекснылм $H$-ростком Маслова $\check{r}(M)$ - множество всех таких $\chi \in \mathscr{H}_{\mathbb{R} C}, \chi\langle\perp\rangle \mathscr{L}_{k}$, что $\Omega[\chi] \Psi_{M} \sim 0$ (равенство нулю понимается в смысле гильбертова пространства $\bar{S})$.

Оказьвается, что $r(M)$ является графиком оператора $\widehat{M}$ :

$$
r(M)=\left\{\left(\begin{array}{c}
\widehat{M} \varphi^{*} \\
\varphi^{*}
\end{array}\right), \varphi^{*} \in \mathscr{H}^{*}\right\}
$$

Обозначим через $r_{\perp}(M)$ множество всех векторов $r(M)$, косоортогональных $\mathscr{L}_{k}^{\mathbb{C}}$. 
ТЕОРема 4. 1) Комплексный Н-росток Маслова $\check{r}(M)$ представляется в виде прямой суммы $\check{r}(M)=r_{\perp}(M) \oplus \mathscr{L}_{k}^{\mathbb{C}}$.

2) $\check{r}=\check{r}(M)$ удовлетворяет следующим свойствам:

(a) $\chi \in \mathscr{L}_{k}^{\mathbb{C}} \Rightarrow \chi \in \check{r}, \frac{1}{i}\left\langle\chi, \chi^{*}\right\rangle=0$;

(б) $\chi \in \check{r}, \chi \notin \mathscr{L}_{k}^{\mathbb{C}} \Rightarrow \frac{1}{i}\left\langle\chi, \chi^{*}\right\rangle>0$;

(в) $\chi_{1}, \chi_{2} \in \check{r} \Rightarrow\left\langle\chi_{1}, \chi_{2}\right\rangle=0$;

(г) любой әлемент $\chi \in \mathscr{H}_{\mathbb{R C}}$, косоортогональный $\mathscr{L}_{k}^{\mathbb{C}}$, представляется в виде

$$
\chi=\chi_{+}+\chi_{-}, \quad \chi_{-} \in \check{r}, \quad \chi_{+} \in \check{r}^{*},
$$

при этом $\chi_{ \pm}$определены однозначно с точностью до добавления әлементов $\mathscr{L}_{k}^{\mathbb{C}}$; определены операторы проектирования

$$
\mathscr{P}: \mathscr{L}_{k}^{\mathbb{C}\langle\perp\rangle} \rightarrow \check{r} / \mathscr{L}_{k}^{\mathbb{C}}, \quad \mathscr{P}^{*}: \mathscr{L}_{k}^{\mathbb{C}\langle\perp\rangle} \rightarrow \check{r}^{*} / \mathscr{L}_{k}^{\mathbb{C}}
$$

вида $\mathscr{P} \chi=\left[\chi_{-}\right], \mathscr{P}^{*} \chi=\left[\chi_{+}\right]$

(д) сужение оператора проектирования $\mathscr{P}$ на

$$
\mathscr{H}=\left\{\left(\begin{array}{l}
f \\
0
\end{array}\right)\right\} \subset \mathscr{H}_{\mathbb{R} C}
$$

является оператором Гильберта-Шмидта из Н̈ в гильбертово пространство $\check{r} / \mathscr{L}_{k}^{\mathbb{C}}$ с нормой $\|\chi\|^{2}=\frac{1}{i}\left\langle\chi, \chi^{*}\right\rangle$.

3) Пусть подпространство г̆ $\subset \mathscr{L}_{k}^{\mathbb{C} \perp \perp\rangle}$ удовлетворяет свойствам (а)-(д) n. 2. Тогда для некоторого гауссовского вектора $\check{r}=\check{r}(M)$.

4) Для двух ненулевых гауссовских векторов $\Psi_{M_{1}} u \Psi_{M_{2}}$ комплексные Н-ростки Маслова совпадают тогда и только тогда, когда $\Psi_{M_{1}} \sim c \Psi_{M_{2}}$ для некоторого числового множителя $c$.

5. Эволюция квантовой системызадается однопараметрической группой $e^{-i H t}$ унитарных операторов, действующих в гильбертовом пространстве. Генератор группы $H$ назьвается гамильтонианом. В приложениях часто встречается случай гамильтониана, квадратичного по операторам рождения и уничтожения:

$$
H=\frac{1}{2} a^{+} A a^{+}+a^{+} \widehat{B} a^{-}+\frac{1}{2} a^{-} A^{*} a^{-}+\varepsilon .
$$

Пусть $\mathscr{L}_{k} \subset D(\widehat{B}), A \in D(\widehat{B}) \otimes D(\widehat{B})$, а отображение $L: \mathscr{H}_{\mathbb{R}} \rightarrow \mathscr{H}_{\mathbb{R}}$ вида

$$
L\left(\begin{array}{c}
\varphi \\
\varphi^{*}
\end{array}\right)=\left(\begin{array}{c}
-i \widehat{B} \varphi-i \widehat{A} \varphi^{*} \\
i \widehat{A}^{*} \varphi+i(\widehat{B} \varphi)^{*}
\end{array}\right),
$$

удовлетворяет свойству $L \mathscr{L}_{k} \subset \mathscr{L}_{k}$. Предположим также, что

$$
\operatorname{Im} \varepsilon=-\frac{1}{2} \operatorname{Tr} L_{0}
$$

где $L_{0}$ - сужение оператора $L$ на $\mathscr{L}_{k}$.

Tеорема 5. 1) Ha $S \cap D\left(a^{+} \widehat{B} a^{-}\right)$оператор $H$ является существенно самосопряженным. При $M \in D(\widehat{B}) \otimes D(\widehat{B})$ u $\chi_{1}, \ldots, \chi_{p} \in D(\widehat{B})$ вектор $\Omega\left[\chi_{1}\right] \ldots \Omega\left[\chi_{p}\right] \Psi_{M}$ принадлеxum $D(H)$.

2) Пусть

$$
\chi(t)=\left(\begin{array}{c}
f(t) \\
\varphi^{*}(t)
\end{array}\right)
$$

удовлетворяет уравнению

$$
\frac{d}{d t} \chi(t)=L^{\mathbb{C}} \chi(t)
$$


Тогда оператор $\Omega[\chi(t)]$ коммутирует с $i \frac{d}{d t}-H$. При $\varphi_{0}, f_{0} \in D(\widehat{B})$ существует единственное решение задачи Коши для уравнения (6). Разрешающий оператор

$$
u_{t}: \chi_{0}=\left(\begin{array}{c}
f_{0} \\
\varphi_{0}^{*}
\end{array}\right) \mapsto \chi(t)
$$

ограничен и однозначно продолжается на $\mathscr{H}_{\mathbb{R C}}$. Отображение $u_{t}=e^{L t}$ сохраняет кососкалярное произведение и удовлетворяет свойству $u_{t} \mathscr{L}_{k}=\mathscr{L}_{k}$.

6. В конечномерном случае для систем без связей известна теорема Маслова [5] об устойчивости классической системы уравнений в случае существования гауссовской собственной функции у квадратичного оператора Гамильтона. Приведем обобщение этой теоремы на бесконечномерный случай и на системы со связями.

Рассмотрим сужение отображения $u_{t}$ на факторпространство $\mathscr{L}_{k}^{\langle\perp\rangle} / \mathscr{L}_{k}:$ ввиду свойства $u_{t} \mathscr{L}_{k}=\mathscr{L}_{k}$ и сохранения кососкалярного произведения такое сужение корректно. На факторпространстве введем норму $\|[\chi]\|=\inf _{\chi \in[\chi]}\|\chi\|$.

Tеорема 6. Пусть оператор $H$ имеет гауссовскую собственную функиию (4) при $M \in$ $D(\widehat{B})$. Тогда классическая система

$$
\frac{d \chi}{d t}=L \chi
$$

устойчива в топологии факторпространства $\mathscr{L}_{k}^{\langle\perp\rangle} / \mathscr{L}_{k}:$ функиия $\left\|u_{t} \chi_{0}\right\|$ ограничена равномерно по $t$ при любом $\chi \in \mathscr{L}_{k}^{\langle\perp\rangle} / \mathscr{L}_{k}$.

ЗАмечАниЕ. Выбор топологии факторпространства является существенным: решение системы (7), рассматриваемое в обычной топологии $\mathscr{H}_{\mathbb{R}}$, может оказаться растущим по $t$.

Приведем схему доказательства теоремы 6 . Пусть $H \Psi_{M}=\varepsilon_{0} \Psi_{M}$. Тогда норма вектора

$$
e^{-i H t} \Omega[\chi] \Psi_{M}=\Omega\left[u_{t} \chi\right] e^{-i \varepsilon_{0} t} \Psi_{M}
$$

должна сохраняться во времени при любом $\chi \in \mathscr{L}_{k}^{\langle\perp\rangle}$. Но вектор (8) можно представить согласно п. (г) теоремы 4 как

$$
e^{-i \varepsilon_{0} t}\left[\Omega\left[\mathscr{P}_{+} u_{t} \chi\right] \Psi_{M}+\Omega\left[\mathscr{P}_{-} u_{t} \chi\right] \Psi_{M}\right]=e^{-i \varepsilon_{0} t} \Omega\left[\mathscr{P}_{+} u_{t} \chi\right] \Psi_{M}
$$

и сохраняющийся квадрат его нормы равен

$$
\frac{1}{i}\left\langle\left(\Omega\left[\mathscr{P}_{+} u_{t} \chi\right] \Psi_{M}\right)^{*}, \Omega\left[\mathscr{P}_{+} u_{t} \chi\right] \Psi_{M}\right\rangle=\left\|\Omega\left[\mathscr{P}_{-} u_{t} \chi\right] \Psi_{M}\right\|^{2} .
$$

Остается проверить, что оператор $\mathscr{P}_{-}: \mathscr{L}_{k}^{\langle\perp\rangle} / \mathscr{L}_{k} \rightarrow \check{r} / \mathscr{L}_{k}^{\mathbb{C}}$, понимаемый как отображение вещественных гильбертовых пространств, обладает ограниченным обратным. Предположим противное. Тогда для некоторой последовательности $\chi_{n} \in \mathscr{L}_{k}^{\langle\perp\rangle} / \mathscr{L}_{k}$ с единичной нормой $\left\|\chi_{n}\right\|=$ $\left\|\mathscr{P}_{-} \chi_{n}+\left(\mathscr{P}_{-} \chi_{n}\right)^{*}\right\|=1$ справедливо свойство $\left\|\mathscr{P}_{-} \chi_{n}\right\|_{\check{r}} \rightarrow 0$. При

$$
\chi_{n}=\left(\begin{array}{c}
f_{n} \\
\varphi_{n}^{*}
\end{array}\right)
$$

эти свойства означают $\left\|f_{n}+\varphi_{n}\right\|_{\mathscr{H}_{\mathbb{R}}}=1,\left\|\varphi_{n}\right\|^{2}-\left\|f_{n}\right\|^{2} \rightarrow 0$. Но $\left\|f_{n}\right\| \leqslant\|\widehat{M}\| \cdot\left\|\varphi_{n}\right\|,\|\widehat{M}\|<1$, поэтому отсюда вытекает, что $\left\|\varphi_{n}\right\| \rightarrow 0,\left\|\varphi_{n}\right\| \rightarrow 0$. Полученное противоречие доказьвает теорему. 


\section{СПИСОК ЦИТИРОВАННОЙ ЛИТЕРАТУРЫ}

1. Славнов А.А., Фаддеев Л. Д. Введение в квантовую теорию калибровочных полей. M.: Наука, 1988. 2. Ashtekar A. et al. // J. Math. Phys. 1995. V. 36. №11. P. 6456-6493. 3. Бeрезин Ф. А. Метод вторичного квантования. М.: Наука, 1986. 4. Маслов В. П. Операторные методы. М.: Наука, 1973. 5. Маслов В. П. Комплексньй метод ВКБ в нелинейньгх уравнениях. М.: Наука, 1977.

Московский государственный университет им. М. В. Ломоносова E-mail: shvedov@qs.phys.msu.su 